\title{
Control design using backstepping technique for a cart-inverted pendulum system
}

\author{
Tran Thien Dung, Nguyen Nam Trung, Nguyen Van Lanh
}

\begin{abstract}
The cart-inverted pendulum system is one of the classical experimental systems that fully converges the complex properties of nonlinear control problems. It represents a class of real world systems such as two-wheeled mobile robots, pendubots, missile launchers and many more. The problems associated with it are always challenging topics in the field of control systems. This paper presents a novel technique to control this system stabilizing at a vertical upright position, its unstable equilibrium point. Simulation and experimental results will show a better performance of the proposed controller in comparison with Quadratic Optimal Regulator method under disturbance and change in mass.
\end{abstract}

Index Terms - Cart-inverted pendulum, Backstepping, DC motor, Quadratic Optimal Regulator.

\section{INTRODUCTION}

The cart-inverted pendulum system has two equilibrium points [1], [8] the stable point is at which the pendulum is pointing downwards and the unstable one is at which the pendulum is pointing upwards. The aim of designing a controller is to move and balance the pendulum from the stable equilibrium point to the unstable one. This is a challenging control problem because the system is highly unstable, nonlinear and underactuated. Different control agorithms are studied by many researchers, from classical PID controllers [2], [13] to advanced controllers such as fuzzy control [3], [14] neural networks [4], [15] and genetic algorithms [5], [16]. Recently, optimal control approach is one of the potential solutions for a given set of performance objectives [17], [21], with detail review in [6]. In [7] and [20], state space control using Linear Quadratic Regulator (LQR) is presented and successfully conducted.

The goal of this article is to design controllers to swing up and balance the pendulum from a pending position to the vertical upward point. Swinging up the pendulum can be achieved by using an energy control [8], [18], [22]. At the vertical position, another controller is used to stabilize the pendulum. In this paper, a stabilizing controller based on backstepping technique [9], [10], [19], is designed and compared to the Quadratic optimal controller [11], [12], [23]. A switch is used to change controllers. This means, when the pendulum approaches a certain area, the stabilizing controller

Tran Thien Dung, Falcuty of Electronics Engineering, Thai Nguyen University of Technology, Vietnam.

Nguyen Nam Trung, Falcuty of Electronics Engineering, Thai Nguyen University of Technology, Vietnam.

Nguyen Van Lanh, Falcuty of International Training, Thai Nguyen University of Technology, Vietnam will replaces the swinging up controller to balance the pendulum at the vertical upward position.

The paper is organized as follows. System model is provided in section II, including nonlinear dynamic model of the system, linearized model in state-space form and permanent magnet DC motor dynamics. Section III presents controller design. Then, section IV shows simulation and experimental results. Finally, Section 5 concludes this paper.

\section{SYSTEM MODELS}

\section{A. Nonlinear Dynamic Model}

In our research, the model of inverted pendulum system is pre-designed and simulated on 3D Solidworks software. Then, an experimental setup is built as shown in Fig. 1. The setup consists of a movable cart driven by a DC motor according to the control voltage. The cart can move along a horizontal track. A pendulum is mounted on the cart and can freely rotate around its axis.

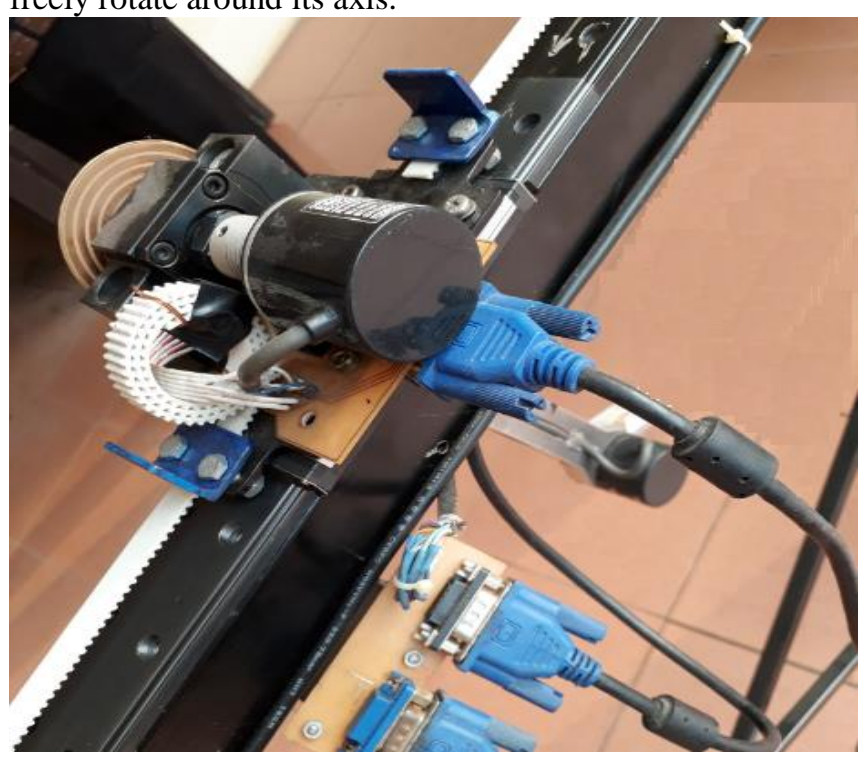

Fig. 1: Snapshot of Real plant

The inverted pendulum is an open-loop, unstable and highly nonlinear system. The objective of the controller is to balance the pendulum at its upward position. Parameters of the system are showed in table 1 .

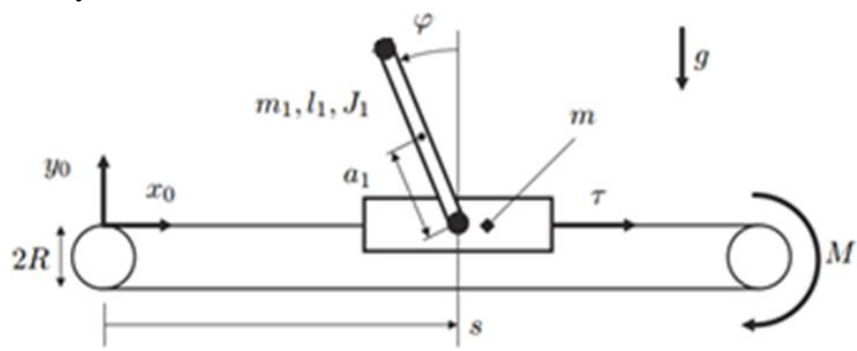

Fig. 2: Reference frames and parameters of pendulum 
Figure 2 shows the reference frames and parameters of the system. The movement of the cart is constrained in the $x$-horizontal direction, and the pendulum can rotate in the $x-y$ plane. The system has two DOF and can be fully represented using two coordinates: horizontal displacement of the cart, $s$; and rotational displacement of pendulum, $\varphi$. Coordinates of the Centre of Gravity ( $\mathrm{CoG}$ ) of the pendulum is given by:

$$
\begin{aligned}
& \mathbf{c}_{1}=\left[\begin{array}{lll}
s-a_{1} \sin \varphi & a_{1} \cos \varphi & 0
\end{array}\right]^{T} \\
& \dot{\mathbf{c}}_{1}=\left[\begin{array}{lll}
s-a_{1} \dot{\varphi} \cos \varphi & -a_{1} \dot{\varphi} \sin \varphi & 0
\end{array}\right]^{T}
\end{aligned}
$$

Table 1: Parameters of the inverted pendulum

\begin{tabular}{|c|c|l|}
\hline Variable & Unit & \multicolumn{1}{|c|}{ Meaning } \\
\hline$s$ & $r a d$ & $\begin{array}{l}\text { Angular displacement of the } \\
\text { pendulum from the vertical upright } \\
\text { position. }\end{array}$ \\
\hline$J_{1}$ & $k g \cdot m^{2}$ & Moment of inertia of the pendulum. \\
\hline$m_{1}$ & $k g$ & Mass of the pendulum. \\
\hline$m$ & $k g$ & The mass of the cart \\
\hline$a_{1}$ & $m$ & $\begin{array}{l}\text { The distance from the CoG of the } \\
\text { pendulum to the pivot. }\end{array}$ \\
\hline$g$ & $m / s^{2}$ & Acceleration of gravity \\
\hline$d_{0}$ & $N m s$ & Friction coefficient with the rail \\
\hline$d_{1}$ & $N m s$ & Friction coefficient of pendulum \\
\hline$R_{m}$ & $\Omega$ & Armature resistance of motor \\
\hline$L_{m}$ & $H$ & Armature inductance of motor \\
\hline$K_{m}$ & $\mathrm{~Wb}$ & Emf constant \\
\hline$R$ & $m$ & Pully radius \\
\hline & & \\
\hline
\end{tabular}

Applying Euler-Lagrangian equation to the system yields:

$$
\frac{d}{d t}\left(\frac{\partial L}{\partial \dot{q}}\right)-\frac{\partial L}{\partial q}=F-\frac{\partial R}{\partial \dot{q}}
$$

where $\mathrm{L}$ is the Lagrange function defined as the difference between kinetic $(\mathrm{T})$ and potential $(\mathrm{V})$ energies: $\mathrm{L}=\mathrm{T}-\mathrm{V}$.

$$
\begin{aligned}
& T=\frac{1}{2} m \dot{s}^{2}+\frac{1}{2} m_{1}\left[\left(\dot{s}-a_{1} \dot{\varphi} \cos \varphi\right)^{2}+a_{1}^{2} \dot{\varphi}^{2} \sin ^{2} \varphi\right]+\frac{1}{2} J_{1} \dot{\varphi}^{2}, \\
& V=m_{1} g a_{1} \cos \varphi ; \quad R=\frac{1}{2}\left(d_{1}+d_{0}\right) \dot{q} \\
& q=\left[\begin{array}{ll}
\varphi & \mathrm{s}
\end{array}\right]^{T} ; \quad F=\left[\begin{array}{ll}
0 & \tau
\end{array}\right]^{T}, \quad \tau=M / R \\
& \frac{\partial L}{\partial \dot{\varphi}}=\left(J+m_{1} a_{11}^{2}\right) \dot{\varphi}+\left(-m_{1} a_{1} \cos \varphi\right) \dot{s} \\
& \frac{d}{d t}\left(\frac{\partial L}{\partial \dot{\varphi}}\right)=\left(J_{1}+m_{1} a_{1}^{2}\right) \ddot{\varphi}+\left(-m_{1} a_{1} \cos \varphi\right) \ddot{s}+\left(m_{1} a_{1} \dot{\varphi} \sin \varphi\right) \dot{s} \\
& \frac{\partial L}{\partial \dot{s}}=\left(-m_{1} a_{1} \cos \varphi\right) \dot{\varphi}+\left(\mathrm{m}+m_{1}\right) \dot{\mathrm{s}} \\
& \frac{d}{d t}\left(\frac{\partial L}{\partial \dot{s}}\right)=\left(-\mathrm{m}_{1} \mathrm{a}_{1} \cos \varphi\right) \ddot{\varphi}+\left(\mathrm{m}+\mathrm{m}_{1}\right) \ddot{\mathrm{s}}+m_{1} a_{1} \dot{\varphi}^{2} \sin \varphi \\
& \frac{\partial L}{\partial \varphi}=\left(m_{1} a_{1} \omega \sin \varphi\right) \dot{s}+m_{1} \mathrm{a}_{1} \mathrm{~g} \sin \varphi ; \quad \frac{\partial L}{\partial s}=0 \\
& \left\{\left(\mathrm{~J}_{1}+\mathrm{m}_{1} \mathrm{a}_{1}^{2}\right) \ddot{\varphi}+\left(-\mathrm{m}_{1} \mathrm{a}_{1} \cos \varphi\right) \ddot{\mathrm{s}}-m_{1} \mathrm{a}_{1} g \sin \varphi+d_{1} \dot{\varphi}=0\right. \\
& \left\{\left(-m_{1} a_{1} \cos \varphi\right) \ddot{\varphi}+\left(m+m_{1}\right) \ddot{s}+\mathrm{m}_{1} \mathrm{a}_{1} \dot{\varphi}^{2} \sin \varphi+d_{0} \dot{s}=\tau\right. \\
& \mathbf{D}(\mathrm{q}) \ddot{\mathrm{q}}+\mathbf{C}(q, \dot{q}) \dot{q}+\mathbf{G} \dot{q}+\mathbf{g}(q)=F
\end{aligned}
$$

$$
\begin{gathered}
\mathbf{D}(\mathrm{q})=\left[\begin{array}{cc}
J_{1}+m_{1} a_{1}^{2} & -m_{1} a_{1} \cos \varphi \\
-m_{1} a_{1} \cos \varphi & m+m_{1}
\end{array}\right] ; \quad \mathbf{C}(q, \dot{q})=\left[\begin{array}{cc}
0 & 0 \\
m_{1} a_{1} \dot{\varphi} \sin \varphi & 0
\end{array}\right] ; \\
\mathbf{G}=\left[\begin{array}{cc}
d_{1} & 0 \\
0 & d_{0}
\end{array}\right] ; \quad \mathbf{g}(q)=\left[\begin{array}{c}
-m_{1} a_{1} g \sin \varphi \\
0
\end{array}\right] ; \\
\ddot{q}=\frac{1}{\mathbf{D}(\mathrm{q})}[F-\mathbf{C}(q, \dot{q}) \dot{q}-\mathbf{G} \dot{q}-\mathbf{g}(q)] \\
\ddot{q}=\left[\begin{array}{l}
\frac{\left(-m-m_{1}\right)\left(-d_{1} \dot{\varphi}+m_{1} a_{1} g \sin \varphi\right)}{m_{1}^{2} a_{1}^{2} \cos ^{2} \varphi-\left(\mathrm{J}_{1}+m_{1} a_{1}^{2}\right)\left(\mathrm{m}+\mathrm{m}_{1}\right)}+\frac{m_{1} a_{1} \cos \varphi\left(-m_{1} a_{1} \dot{\varphi}^{2} \sin \varphi-d_{0} \dot{s}+\frac{M}{R}\right)}{\left(\mathrm{m}+\mathrm{m}_{1}\right)\left(\mathrm{J}_{1}+\mathrm{m}_{1} \mathrm{a}_{1}^{2}\right)-\mathrm{m}_{1}^{2} a_{1}^{2} \cos ^{2} \varphi} \\
\frac{-m_{1} a_{1} \cos \varphi\left(-d_{1} \dot{\varphi}+m_{1} a_{1} g \sin \varphi\right)}{m_{1}^{2} a_{1}^{2} \cos ^{2} \varphi-\left(J_{1}+m_{1} a_{1}^{2}\right)\left(\mathrm{m}+\mathrm{m}_{1}\right)}+\frac{\left(J_{1}+m_{1} a_{1}^{2}\right)\left(-m_{1} a_{1} \dot{\varphi}_{1}^{2} \sin \varphi-d_{0} \dot{s}+\frac{M}{R}\right)}{\left(\mathrm{m}+\mathrm{m}_{1}\right)\left(\mathrm{J}_{1}+\mathrm{m}_{1} \mathrm{a}_{1}^{2}\right)-\mathrm{m}_{1}^{2} a_{1}^{2} \cos ^{2} \varphi}
\end{array}\right]
\end{gathered}
$$

Linearizing the model, the following approximations are applied: $\varphi \approx 0 \Rightarrow \sin \varphi \approx \varphi ; \cos \varphi \approx 1$

Defining the state variables as below:

$$
x_{1}=\varphi ; x_{2}=\dot{\varphi} ; x_{3}=s ; x_{4}=\dot{s} ; \quad x=\left[\begin{array}{llll}
x_{1} & x_{2} & x_{3} & x_{4}
\end{array}\right]^{T}
$$

The linearized model, thus, becomes:

$$
\left\{\begin{array}{l}
\ddot{x}_{1}=\frac{\left(m+m_{1}\right)\left(-d_{1} \mathrm{x}_{2}+m_{1} \mathrm{a}_{1} \mathrm{gx}_{1}\right)+\mathrm{m}_{1} a_{1}\left(\frac{M}{R}-\mathrm{d}_{0} \mathrm{x}_{4}\right)}{\left(\mathrm{J}_{1}+m_{1} a_{1}^{2}\right)\left(\mathrm{m}+\mathrm{m}_{1}\right)-m_{1}^{2} a_{1}^{2}} \\
\ddot{x}_{3}=\frac{m_{1} a_{1}\left(-\mathrm{d}_{1} \mathrm{x}_{2}+\mathrm{m}_{1} \mathrm{a}_{1} \mathrm{gx}_{1}\right)+\left(\mathrm{J}_{1}+\mathrm{m}_{1} \mathrm{a}_{1}^{2}\right)\left(\frac{M}{R}-\mathrm{d}_{0} \mathrm{x}_{4}\right)}{\left(J_{1}+m_{1} a_{1}^{2}\right)\left(\mathrm{m}+\mathrm{m}_{1}\right)-m_{1}^{2} a_{1}^{2}}
\end{array}\right.
$$

B. Linearized Model in State-Space Form

Linearizing the inverted pendulum system results in:

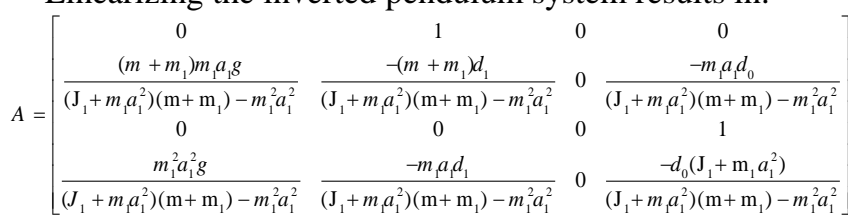

$$
B=\left[\begin{array}{c}
0 \\
\frac{m_{1} a_{1}}{\left(\mathrm{~J}_{1}+m_{1} a_{1}^{2}\right)\left(\mathrm{m}+\mathrm{m}_{1}\right)-m_{1}^{2} a_{1}^{2}} \\
0 \\
\frac{\left(J_{1}+m_{1} a_{1}^{2}\right)}{\left(\mathrm{J}_{1}+m_{1} a_{1}^{2}\right)\left(\mathrm{m}+\mathrm{m}_{1}\right)-m_{1}^{2} a_{1}^{2}}
\end{array}\right]
$$

Table 2: List of Parameters

\begin{tabular}{|c|c|c|}
\hline Variable & Value & Unit \\
\hline$J_{1}$ & 0.0052 & $\mathrm{~kg} \cdot \mathrm{m}^{2}$ \\
\hline$m_{1}$ & 0.43 & $\mathrm{~kg}$ \\
\hline$m$ & 1.3 & $\mathrm{~kg}$ \\
\hline$a_{1}$ & 0.157 & $\mathrm{~m}$ \\
\hline$g$ & 9.81 & $\mathrm{~m} / \mathrm{s}^{2}$ \\
\hline$d_{0}$ & 0.147 & $\mathrm{Nm} . \mathrm{s}$ \\
\hline$d_{1}$ & 0.00243 & $\mathrm{Nm} . \mathrm{s}$ \\
\hline
\end{tabular}

Substituting the parameters given in Table 1 into (4), we obtain: 
$\left\{\begin{array}{l}\dot{x}=A x+B u \\ y=C x\end{array} ; x=\left[\begin{array}{llll}x_{1} & x_{2} & x_{3} & x_{4}\end{array}\right]^{T} ; y=\left[\begin{array}{ll}x_{1} & x_{3}\end{array}\right]^{T}\right.$

$A=\left[\begin{array}{cccc}0 & 1 & 0 & 0 \\ 50.307 & -0.1846 & 0 & -0.4357 \\ 0 & 0 & 0 & 1 \\ 1.9631 & -0.007203 & 0 & -0.102\end{array}\right] \quad B=\left[\begin{array}{c}0 \\ 2.9642 \\ 0 \\ 0.6937\end{array}\right]$

\section{Permanent Magnet DC Motor Dynamics}

The relation between the armature current and the armature voltage can be written in Laplace form as:

$$
U_{m}=E_{\mathrm{emf}}+I_{m}\left(R_{m}+s L_{m}\right)
$$

where $R_{\mathrm{m}}$ and $L_{\mathrm{m}}$ are resistance and inductance of the rotor, respectively.

The back-emf voltage created by the motor, $\mathrm{E}_{\mathrm{emf}}$, is proportional to the rotor speed as:

$$
E_{\text {emf }}=K_{m} \dot{\varphi}
$$

The electromagnetic torque generated by the DC motor is proportional to the armature current:

$$
M_{d t}=K_{m} I_{m}
$$

We have:

$$
I_{m}=\frac{1}{R_{m}+L_{m} s}\left(U-E_{e m f}\right)=\frac{1 / R_{m}}{T_{m} s+1}\left(U-K_{m} \dot{\varphi}\right)
$$

From the above equations, we get the structure diagram of DC motor with feedback current using ACS 712 current sensor as follows:

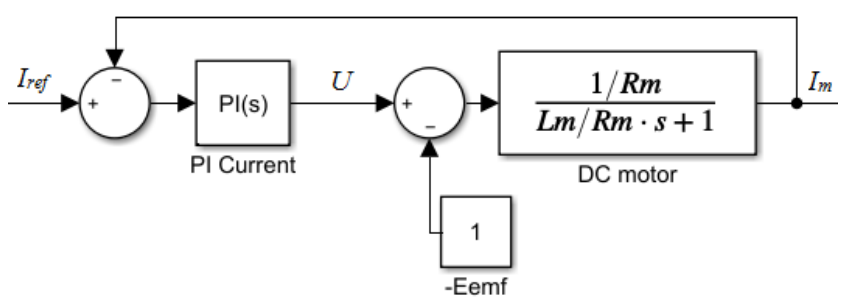

Fig. 3: Closed-Loop DC motor current Control System

The response rate of the current controller is very fast, so the change from the feedback output is very small. Therefore, the feedback is considered as a noise.

Table 3: List of Parameters.

\begin{tabular}{|c|c|}
\hline Parameter & Value \\
\hline DC motor power $(P)$ & $120 \mathrm{~W}$ \\
\hline voltage $(U)$ & 24 VDC \\
\hline Current $(I)$ & $5 \mathrm{~A}$ \\
\hline DC motor speed $(n)$ & $1200 R p m$ \\
\hline rotor inertia $\left(J_{D}\right)$ & $2.10^{-4} \mathrm{Kg} \cdot \mathrm{m}^{2}$ \\
\hline pully radius $(R)$ & $0.195 \mathrm{~m}$ \\
\hline Armature inductance of motor $\left(L_{m}\right)$ & $0.0281 \mathrm{H}$ \\
\hline Armature resistance of motor $\left(R_{m}\right)$ & $0.34 \Omega$ \\
\hline
\end{tabular}

In the classical sense, a PI controller has the following transfer function:

$$
\mathrm{W}_{c}=K_{p}\left(1+K_{i} \frac{1}{s}\right)=0.32\left(1+660.16 \frac{1}{s}\right)
$$

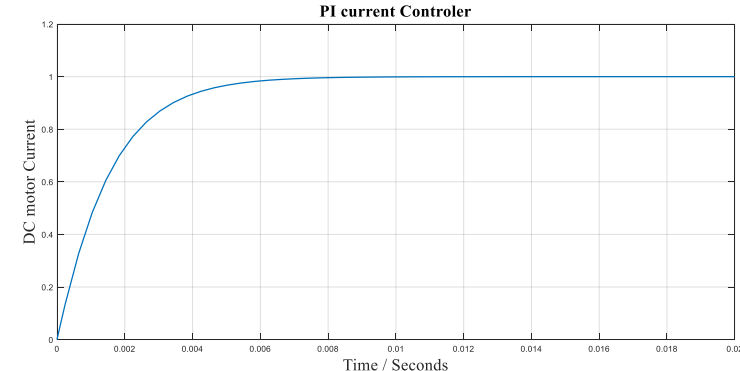

Fig 4: Diagram simulating the current controller with the reference set point to 1

The inner loop needs a fast response. Using PI controller with the above parameters, the system has a Settling Time of $0.008 \mathrm{~s}$. Therefore, the designed PI controller meets the requirement.

\section{DESIGN OF CONTROLLERS}

\section{A. Design and Simulation of inverted pendulum Quadratic} optimal regulator problem

The system equation in the state space is represented as

$$
\left\{\begin{array}{l}
\dot{x}=A x+B u \\
y=C x
\end{array}\right.
$$

We determine the matrix $\mathrm{K}$ of the optimal control vector $u=-K x$ to minimize the performance index:

$$
\mathrm{J}=\frac{1}{2} \int_{0}^{+\infty}\left(\mathrm{x}^{\mathrm{T}} \mathrm{Qx}+\mathrm{u}^{\mathrm{T}} \mathrm{Ru}\right) \mathrm{dt} \rightarrow \min
$$

Where $\mathrm{Q}$ and $\mathrm{R}$ are weighting matrices. In this problem, we assume that the control vector $\mathrm{u}(\mathrm{t})$ is unconstrained. The linear control law given by Eq. (8) is the optimal control law. The matrix $\mathrm{K}$ are determined by minimizing the performance index $J$, then $\mathrm{u}(\mathrm{t})=-\mathrm{Kx}(\mathrm{t})$ is the optimal control signal for any initial state $\mathrm{x}(0)$. The block diagram is shown in Fig 4 .

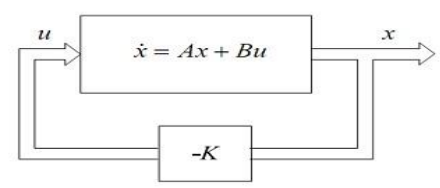

Fig. 5: Block diagram of the optimal regulator system

$$
A=\left[\begin{array}{cccc}
0 & 1 & 0 & 0 \\
50.307 & -0.1846 & 0 & -0.4357 \\
0 & 0 & 0 & 1 \\
1.9631 & -0.007203 & 0 & -0.102
\end{array}\right] \quad B=\left[\begin{array}{c}
0 \\
2.9642 \\
0 \\
0.6937
\end{array}\right]
$$

In MATLAB, function "lqr" is used to get the corresponding feedback gain matrix $\mathrm{K}=\operatorname{lqr}(\mathrm{A}, \mathrm{B}, \mathrm{Q}, \mathrm{R})$, where $Q$ is a positive semi-definite real symmetric matrix, $R$ is a positive definite real symmetric matrix. $Q$ and $R$ are selected by experience.

$$
\begin{aligned}
& \mathrm{Q}=\operatorname{diag}([5,1,500,1]) \text { and } \mathrm{R}=1 \\
& \mathrm{~K}=\operatorname{lqr}(\mathrm{A}, \mathrm{B}, \mathrm{Q}, \mathrm{R})
\end{aligned}
$$

Resulting in the optimal gain:

$$
K=\left[\begin{array}{llll}
69.5225 & 10.2055 & -22.3607 & -15.6577
\end{array}\right]
$$

\section{B. Backstepping linear design}

The new control variables are defined as: $z_{1}=x_{1}-k_{1} x_{3}$ where $k_{l}$ is a design constant, and $\dot{z}_{1}=x_{2}-k_{1} x_{4}$. Define $x_{2}$ 
as the virtual control variable, for which the stabilizing function is chosen: $\alpha_{1}=-c_{1} z_{1}+k_{1} x_{4}$ where $c_{1}$ is positive. In addition, the corresponding error state variable is defined as $z_{2}=x_{2}-\alpha_{1}$. So, we have: $\dot{z}_{1}=x_{2}-k_{1} x_{4}=z_{2}-c_{1} z_{1}$. The derivative of $z_{2}$ is computed as follows: $\dot{z}_{2}=\dot{x}_{2}-\dot{\alpha}_{1}=\dot{x}_{2}-k_{1} \dot{x}_{4}+c_{1} x_{2}-c_{1} k_{1} x_{4}$.

However, the desired dynamics of $z_{2}$ can be defined:

$$
\dot{z}_{2}=-z_{1}-c_{2} z_{2}
$$

From these above equations, we design a controller as below:

$$
\begin{aligned}
& u=\frac{1}{2.9642-k_{1} 0.6937}\left(h_{1} x_{1}+h_{2} x_{2}+h_{3} x_{3}+h_{4} x_{4}\right) \\
& h_{1}=-51.307+k_{1} 1.9637-c_{1} c_{2} \\
& h_{2}=0.1846-k_{1} 0.007203-\left(c_{1}+c_{2}\right) \\
& h_{3}=k_{1}\left(1+c_{1} c_{2}\right) \\
& h_{4}=0.4357-k_{1}\left(0.102-\left(c_{1}+c_{2}\right)\right)
\end{aligned}
$$

Analyzing stability of the system, we have:

$$
\begin{aligned}
& \dot{V}_{2}=\frac{1}{2} z_{1}^{2}+\frac{1}{2} z_{2}^{2}=z_{1} \dot{z}_{1}+z_{2} \dot{z}_{2}=z_{1}\left(z_{2}-c_{1} z_{1}\right)+ \\
& +z_{2}\left(\dot{x}_{2}-k_{1} \dot{x}_{4}+c_{1} x_{2}-c_{1} k_{1} x_{4}\right)=-c_{1} z_{1}^{2}-c_{2} z_{2}^{2} \leq 0
\end{aligned}
$$

This implies that $z_{1}, z_{2}$ are stable, the state trajectory approaches to the origin, so $x_{1} x_{2}$ are also stable. Note that it is important to choose $k_{1}$ appropriately to stabilize the closed-loop system. This means $k_{1}$ is chosen so that $x_{3}, x_{4}$ are also approaches to zero. As a result, the backstepping controller not only keeps the pendulum at the vertical upright position, but also moves the cart to its original position.

\begin{tabular}{|c|c|c|}
\hline$c_{1}$ & $c_{2}$ & $k_{l}$ \\
\hline 100 & 100 & 0.03 \\
\hline
\end{tabular}

\section{Swing-Up Control}

Neglecting frictions and assuming pendulum as a rigid body, we obtain the equation of motion of the pendulum:

$$
\sin \varphi=\frac{1}{m_{1} a_{1} g}\left(\left(J_{1}+m_{1} a_{1}^{2}\right) \ddot{\varphi}-m_{1} a_{1} \cos \varphi u\right)
$$

We choose the energy of the system as zero in the lower position, and normalize it by $-m g a_{l}$, which is the energy required to raise the pendulum from the hanging down position to the horizontal position. The normalized energy can be then written as below:

$$
E=\frac{1}{2}\left(J_{1}+m_{1} a_{1}^{2}\right) \dot{\varphi}^{2}+m_{1} a_{1} g(\cos \varphi+1)
$$

Computing the derivative of $E$ with respect to time we find: $\dot{E}=\dot{\varphi}\left(\left(J_{1}+m_{1} a_{1}^{2}\right) \ddot{\varphi}-m_{1} a_{1} g \sin \varphi\right)$

$$
\dot{E}=-m_{1} a_{1} g \cdot u \cdot \dot{\varphi} \cos \varphi \quad u=0.117 \ddot{s}
$$

Define the desired energy as $E_{0}=2 m_{1} a_{1} g$. The following control is a strategy for achieving the desired energy

$$
u=-m_{1} a_{1} g(E-E o) \dot{\varphi} \cos \varphi
$$

To change the energy fast, the magnitude of the control signal should be as large as possible. This is achieved with the control law:

$$
u=-k_{z} \cdot \operatorname{sign}((E-E o) \dot{\varphi} \cos \varphi)
$$

where $k_{z}$ is a design parameter.

\section{SIMULATION AND EXPERIMENTAL RESULTS}

\section{A. Simulation results}

Block diagram and simulation result of the controller using swing up in combination with Quadratic optimal control are shown in Figure 6 and Figure 7.

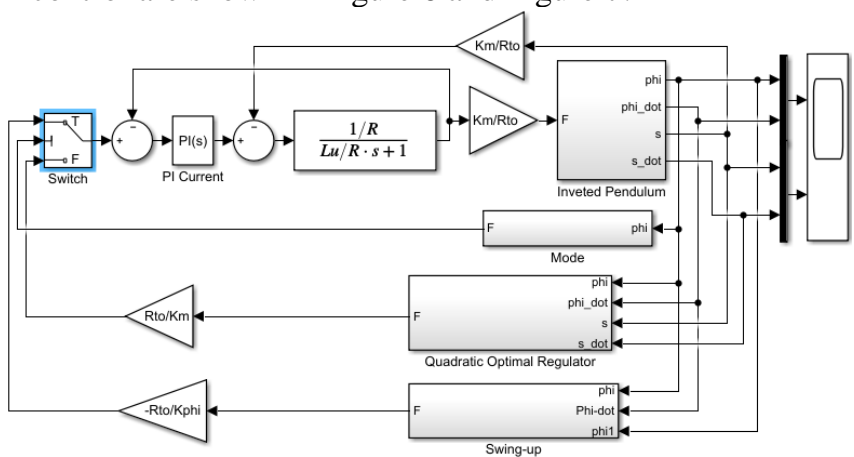

Fig. 6: Block diagram of controllers using Quadratic Optimal Regulator (MATLAB Simulink).

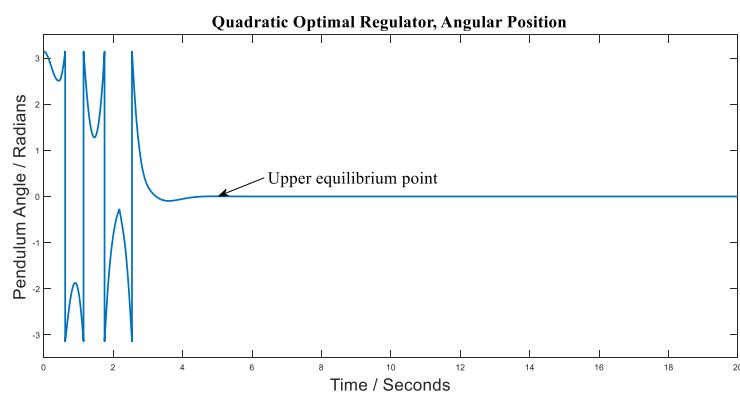

Fig. 7: Simulation of Swing-Up \& Stabilization using Quadratic Optimal Regulator

Block diagram and simulation result of the controller using swing up combined with backstepping control are show in Figure 8 and Figure 9.

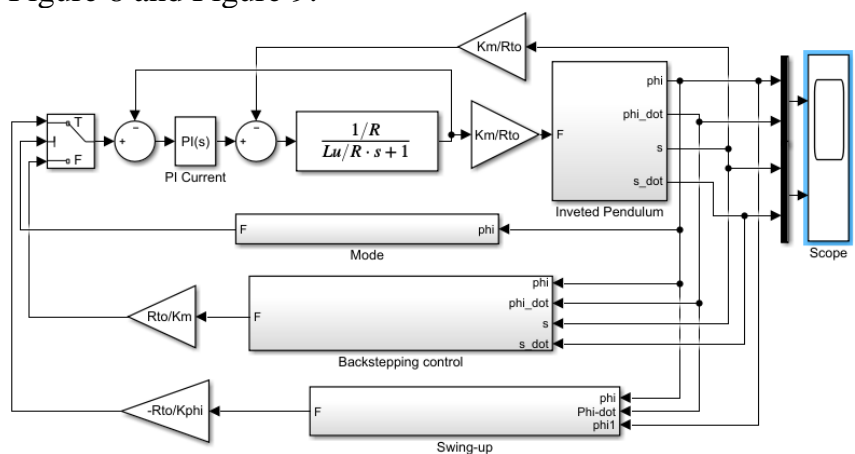

Fig. 8: Block diagram of controller using backstepping control

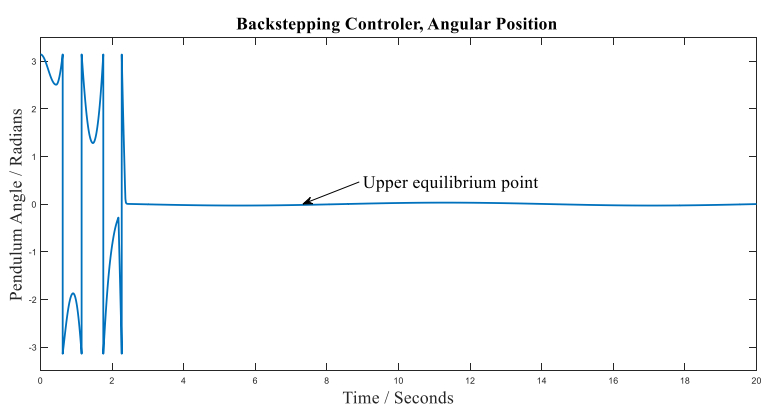

Fig. 9: Simulation of Swing-Up \& Stabilization using Backstepping control 
Fig. 7 and Fig. 9 show that the transition time of the system using Quadratic optimal regulator is nearly 4 seconds, while using backstepping control is only 2.2 seconds. This means that the Backstepping control is much better than the Quadratic optimal regulator.

\section{B. Experimental results}

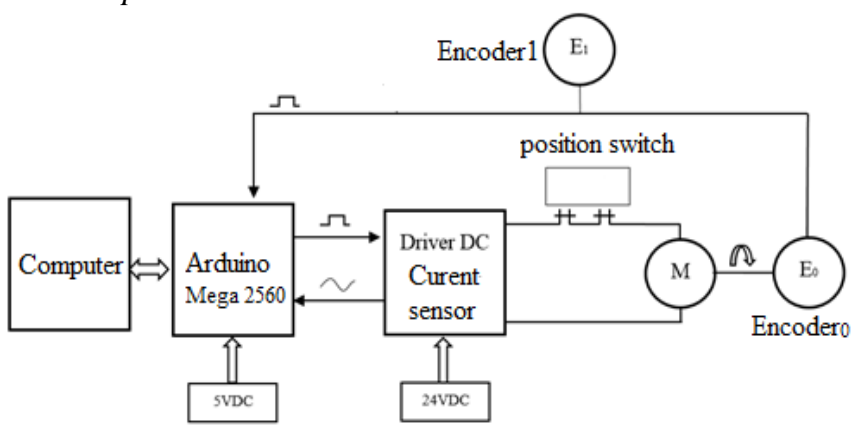

Fig. 10: Block diagram of experimental setup

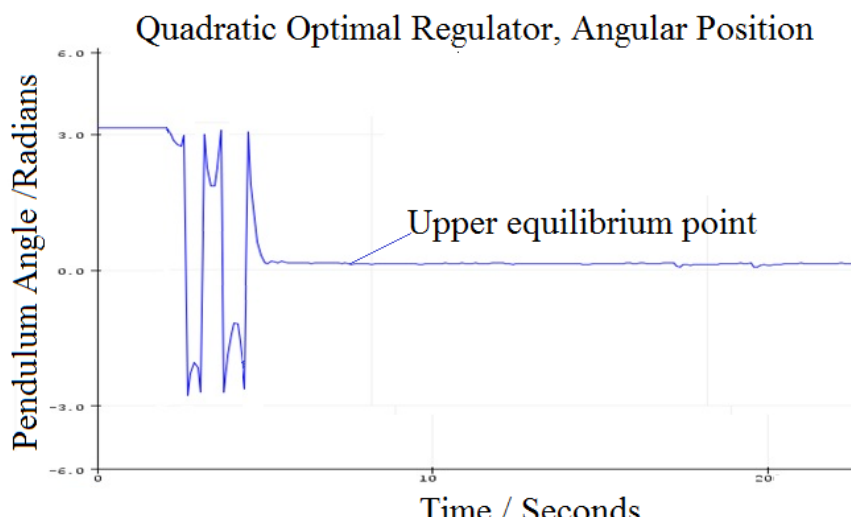

Fig. 11: Experimental Swing-Up \& Stabilization using Quadratic Optimal Regulator

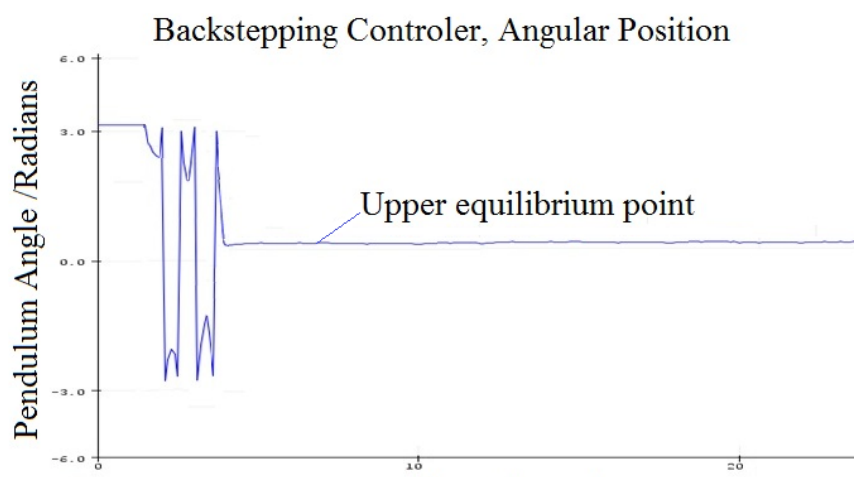

Time / Seconds

Fig. 12: Experimental Swing-Up \& Stabilization using Backstepping control

Figure 10 shows the block diagram of the experimental setup. Experimental results of the controller using swing up combined with Quadratic optimal control in Figure 11 and with the backstepping control in Figure 12. It can be seen that control input $u$ from a combination of a swing up controller and a stabilizing controller is able to move and balance the pendulum from its stable equilibrium point, $x=[\pi, 0,0,0]^{T}$, to its unstable equilibrium point, $x=[0,0,0,0]^{T}$. We also see that the backstepping controller can guarantee a faster and smoother stabilizing process with less oscillation and more robustness than the Quadratic optimal regulator design.

\section{CONCLUSION}

The proposed controller has achieved that the closed-loop system is able not only to swing up and balance the pendulum from downward position to the upward equilibrium point, but also to return the cart to its original position on the rail. The pendulum is stable at its upward position. This proves that the control algorithm is effective. In additions, the performance of controller using backstepping technique is significantly better than that using Quadratic optimal regulator.

Simulation and experimental results are almost similar. In experimental results, however, the pendulum still oscillates slightly around the equilibrium position. This could be due to the dynamic uncertainty, pinion backlash, motor dead-zone, magnetic hysteresis, and other mechanical imperfections. More details about the experiment and its results can be found at: https://m.youtube.com/watch?v=-RfKzVqG2Z0.

Our future research is control design for the triple link inverted pendulum system, as shown in Fig. 13.

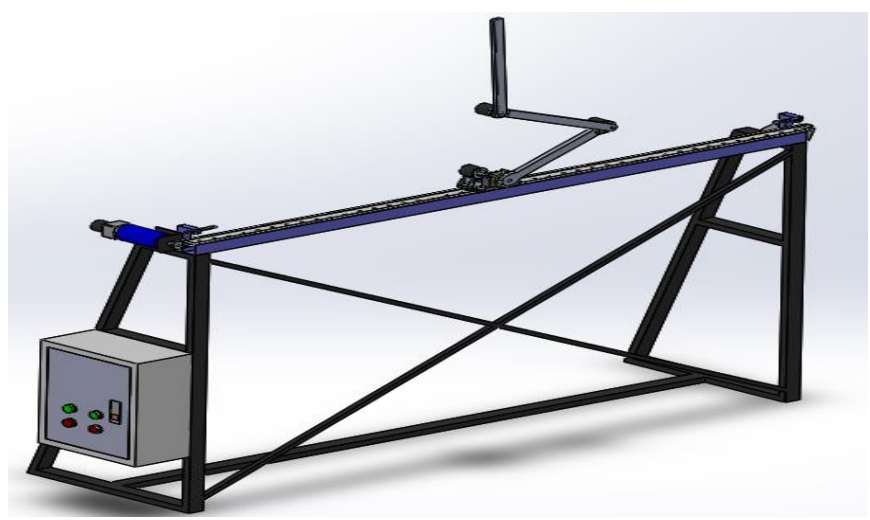

Fig. 13: 3D Solidworks Triple inverted pendulum system

\section{REFERENCES}

[1] R. F. Harrison, "Asymptotically optimal stabilising quadratic control of an inverted pendulum," IEE Proceedings - Control Theory and Applications, vol. 150, no. 1, pp. 7-16, Jan 2003.

[2] C. Wang, G. Yin, C. Liu, and W. Fu, "Design and simulation of inverted pendulum system based on the fractional PID controller," in 2016 IEEE 11th Conference on Industrial Electronics and Applications (ICIEA), June 2016, pp. 1760-1764.

[3] G. O. Tirian, O. Prostean, I. Filip, and C. Rat, "Inverted pendulum controlled through fuzzy logic," in 2015 IEEE 10th Jubilee International Symposium on Applied Computational Intelligence and Informatics, May 2015, pp. 85-90

[4] M. H. Arbo, P. A. Raijmakers, and V. M. Mladenov, "Applications of neural networks for control of a double inverted pendulum," in 12th Symposium on Neural Network Applications in Electrical Engineering (NEUREL), Nov 2014, pp. 89-92.

[5] N. Metni, "Neuro-control of an inverted pendulum using genetic algorithm," in 2009 International Conference on Advances in Computational Tools for Engineering Applications, July 2009, pp. 27-33.

[6] D. Tabak, "Applications of mathematical programming techniques in optimal control: A survey," IEEE Transactions on Automatic Control, vol. 15, no. 6, pp. 688-690, Dec 1970.

[7] Indrazno Siradjuddin, Budhy Setiawan, Ahmad Fahmi, Zakiya Amalia and Erfan Rohadi, "State space control using LQR method for a cart-inverted pendulum linearised model", International Journal of Mechanical \& Mechatronics Engineering IJMME-IJENS Vol:17 No:01.

[8] K.J. Åström, K. Furuta, 2000, "Swinging up a pendulum by energy control", Automatica 36, no.2, pp 287-295.

[9] Rudra S, Barai RK, Maitra M (2014), "Nonlinear state feedback controller design for underactuated mechanical system: a modified block backstepping approach". ISA Trans 53 (2):317-326 
[10] Lixia Deng, Shengxian gGao, 2011, "The design for the controller of the linear inverted pendulum based on backstepping", International Conference on Electronic \& Mechanical Engineering and Information Technology, pp 2892-2895.

[11] Miroslav K., Ioannis K., Petar K. Nonlinear and adaptive control design. Prentice Hall, 1995.

[12] Chandan Kumar, Santosh Lal, Nilanjan Patra, Kaushik Halder, Motahar Reza, "Optimal Controller Design for Inverted Pendulum System based on LQR method", 2012 IEEE International Conference on Advanced Communication Control and Computing Technologies (ICACCCT).

[13] Sandeep D. Hanwate, Yogesh V. Hote, "Design of PID controller for Inverted Pendulum using Stability Boundary Locus", 2014 Annual IEEE India Conference (INDICON).

[14] Ahmad Ilyas Roose, Samer Yahya, Hussain Al-Rizzo, "Fuzzy-logic control of an inverted pendulum on a cart", omputers and Electrical Engineering 61 (2017) 31-47.

[15] Valeri Mladenov, "Application of Neural Networks for Control of Inverted Pendulum", WSEAS Transaction on Circuits and Systems, ISSN: 1109-2734, Issue 2, Volume 10, February 2011.

[16] Tung-Kuan Liu, Yao-Chun Shen and $\mathrm{Zu}$-shu Li, "An Application of Multiobjective Optimization Genetic Algorithm for Cart-Double-Pendulum-System Control”, 2006 IEEE International Conference on Systems, Man, and Cybernetics, October 8-11, 2006, Taipei, Taiwan.

[17] N. Dhang and S. Majumdar, "Optimal Control of Nonlinear Inverted Pendulum System, "Using PID Controller and LQR: Performance Analysis Without and With Disturbance Input", International Journal of Automation and Computing 11(6), December 2014.

[18] Noriko Matsuda, Masaki Izutsu, Jun Ishikawa, Katsuhisa Furuta and Karl J. Astrom, "Swinging-Up and Stabilization Control Based on Natural Frequency for Pendulum Systems", 2009 American Control Conference Hyatt Regency Riverfront, St. Louis, MO, USA June $10-12,2009$.

[19] Rudra S, Barai RK, Maitra M (2014), "Nonlinear state feedback controller design for underactuated mechanical system: a modified block backstepping approach", ISA Trans 53 (2):317-326

[20] Chen, X., Zhou, H., Ma, R \& et al. "Linear Motor Driven Inverted Pendulum and LQR Controller Design", Proceedings of the IEEE International Conference on Automation and Logistics, 2007, pp. 1750-1754.

[21] Merakeb, Abdelkader and Achemine, Farida and Messine, Frédéric Optimal time control to swing-up the inverted pendulum-cart in open-loop form. (2013) In: Electronics, Control, Measurement, Signals and their application to Mechatronics (ECMSM 2013), 24 June 2013 26 June 2013 (Toulouse, France).

[22] W Torres-Pomales, O R Gonzalez, "Nonlinear control of swing-up inverted pendulum," IEEE International Conference on Control Applications, pp. 259-264, SEP. 1996.

[23] Katsuhiko Ogata. Modern Control Engineering. Fifth Edition, Prentice Hall, 2010

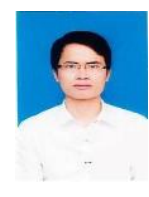

MSc Tran Thien Dung received his bachelor degree of Control Engineering from Thai Nguyen University of Technology (TNUT), Vietnam in 2013, and master degree of Automatic Control from Hanoi University of Science and Technology (HUTS), Vietnam in 2017. He is currently working as a lecturer and researcher at Faculty of Electronics Engineering, TNUT. His research interests are Automation, Control Systems, robotics, adaptive control, sliding mode control, intelligent systems and their industrial applications.

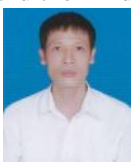

MSc Nguyen Nam Trung obtained his bachelor degree of Electrical Engineering from Thai Nguyen University of Technology (TNUT), Vietnam in 1992, and master degree of Automatic Control from Hanoi University of Science and Technology (HUTS), Vietnam in 2007. Currently he is a lecturer at Faculty of Electronics Engineering, TNUT. His research interests are optimal control, Fuzzy control, motion control, and robotics.

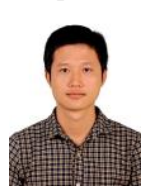

MSc Nguyen Van Lanh received his bachelor degree of Electrical Engineering from Thai Nguyen University of Technology (TNUT), Vietnam in 2011, and Master of Control System Engineering from HAN University of Applied Science, the Netherlands in 2018. He is currently a lecturer and researcher at Faculty of International Training, TNUT. His research interests include learning control systems, optimal control and model predictive controller, nonlinear and adaptive control. 